\title{
PANCREAS AND ISLET TRANSPLANTATION: CLINICAL INDICATIONS AND CLINICAL RESULTS
}

\author{
ANTONIO SECCHI (*) \\ Nota presentata dal m.e. Guido Pozza \\ (Adunanza del 23 ottobre 2014)
}

SunTO. - Il trapianto di pancreas è la cura più efficace in caso di diabete di tipo 1 . Sebbene sia stato dimostrato che uno schema intensivo di somministrazione d'insulina sia in grado di ridurre l'incidenza delle note complicanze del diabete, solo il trapianto di pancreas normalizza il controllo glicometabolico, evita i rischi di gravi episodi d'ipoglicemia e previene $\mathrm{o}$ in alcuni casi migliora le complicanze già riscontrate. Nel seguente articolo sarà analizzato l'impatto del trapianto di pancreas sugli effetti collaterali dovuti a diabete e sulla sopravvivenza dei pazienti. Recentemente è emerso come il trapianto d'isole sia l'approccio terapeutico più promettente per migliorare il controllo glicometabolico nel diabete di tipo 1 e in molti casi per ottenere l'indipendenza da insulina. Il trapianto d'isole richiede inoltre un ricovero ospedaliero relativamente breve e ha il vantaggio di essere una procedura non invasiva. Il grado di insulinoindipendenza ottenuto dopo un anno dal trapianto di isole è migliorato significativamente negli ultimi anni. I dati provenienti da una ricerca internazionale hanno confermato che il trapianto di isole può costituire una cura per il diabete di tipo 1. Dati recenti inoltre indicano che l'insulino indipendenza intervenuta dopo il trapianto di isole si associa a un miglioramento del metabolismo del glucosio e di conseguenza della qualità di vita con una decisa riduzione degli episodi di ipoglicemia. Il trapianto di isole pancreatiche è ancora allo stadio iniziale e quindi è necessario superare altri ostacoli, ma una volta insediato con successo dal punto di vista clinico questo trattamento potrà essere proposto a pazienti diabetici molto prima dell'insorgenza delle complicanze oppure a coloro che rischiano la vita a causa della non consapevolezza della ipoglicemia o nei casi di diabete instabile.

(*) San Raffaele University, Milano, Italy.

E-mail: secchi.antonio@hsr.it 
ABSTRACT. - Pancreas transplantation is the more effective cure for type 1 diabetes. Although intensive insulin scheme has been demonstrated to reduce the incidence of diabetes related complication, only pancreas transplantation normalizes glycometabolic control, avoids the risk of severe hypoglycemic events and prevents or in some case ameliorates diabetes related complications. In this review it will be analyzed the impact of pancreas transplantation on diabetes related complication and patient survival. Islet cell transplantation has recently emerged as one the most promising therapeutic approaches to improving glycometabolic control in type 1 diabetic patients and, in many cases, to obtaining insulin independence. Islet cell transplantation requires a relatively short hospital stay and has the advantage of being a relatively noninvasive procedure. The rate of insulin independence 1 year after islet cell transplantation has significantly improved in recent years Data from a recent international Trial confirmed that islet cell transplantation can be potentially a cure for type 1 diabetes. Recent data indicate that insulin independence after islet cell transplantation is associated with an improvement in glucose metabolism and quality of life and with a reduction in hypoglycemic episodes. Islet cell transplantation is still in its initial stages, and many obstacles still need to be overcome. Once clinical islet transplantation has been successfully established, this treatment could even be offered to diabetic patients long before the onset of diabetic complications or to patients with life-threatening hypoglycemic unawareness and brittle diabetes.

\section{INTRODUCTION}

Pancreas transplant was performed for the first time in 1966 at the University of Minnesota. More than 27,00 pancreas transplants were reported in the USA, and further 15,000 were reported all over the world, reaching at 1 year a survival rate of $95 \%$ for patients and $85 \%$ for pancreas, as reported by the International Pancreas Transplant registry, hold at the University of Arizona [1].

Currently pancreas transplantation represents the preferred treatment for the restoration of the endocrine function, guaranteeing normal glucose homeostasis and consequently the effective reduction of the side effects on the long-term diabetic disease. However, the procedure of pancreas transplant cannot be considered life-saving, in contrast, e.g., to liver or heart /lungs transplant. For this reason, the long-term advantages have to be evaluated and correlated to the potential mortality and morbidity associated with the surgical intervention as well as to the possible side effects due to the chronic immunosuppression therapy.

\section{SELECTION CRITERIA FOR PANCREAS TRANSPLANTATION}

All the patients affected by type 1 diabetes mellitus and end stage renal disease, able to face major surgery, are possible candidates for a 
simultaneous kidney and pancreas transplant. Candidates for isolated pancreas transplant are all the patients suffering type1 diabetes mellitus, who show an elevated risk to develop secondary complications to the disease, such as nephropathy, neuropathy and retinopathy or manifest a severe glycometabolic unbalance, with frequent episodes of undetected hypoglycemia. Particular attention has to be observed in patients who suffer from renal dysfunction: in such case it is very important to consider the risk of progression of disease and consequently the potential necessity to undergo a kidney transplant in the near future.

As a consequence three recipient categories are currently identified:

- simultaneous pancreas kidney transplantation (SPK) in patients affected by type 1 diabetes and end stage renal failure: this represents $80 \%$ of all the pancreas performed.

- Pancreas after kidney transplantation (PAK) in type 1 diabetic patients already submitted to kidney transplantation, either by cadaver or living donors: this represents $15 \%$ of all the transplants performed.

- Pancreas transplant alone (PTA) in brittle diabetic patients: $5-10 \%$ of the cases.

\section{SURGICAL PROCEDURES}

The transplanted pancreas is situated in the right iliac fossa. The blood anastomosis of the transplanted organ are done between the iliac artery of the recipient and the upper mesenteric artery and the splenic artery of the donor. The surgical technique for the venous drainage can be different according to the different receivers: the anastomosis can be prepared on the iliac vein (systemic drain) or on the inferior cava vein or on the mesenteric vein (portal drain). The drainage in the portal system imitates the physiological insulin passage at the hepatic level where it is metabolized by $50 \%$. The systemic vein drain bypasses the effect of the first liver passage, causing hyperinsulinemia, which can predispose and accelerates atherosclerosis [2,3]. Studies have shown how the portal vein drainage, compared with the systemic one, is able to reduce the free cholesterol level, VLDL, LDL and apolipoprotein B [4]. The exocrine secretions are drained into the bladder or at enteric level. The bladder drain is associated with an elevated frequency of urologic side - effects, including urinary tract infections, ematuria, acidosis, dysplasia of the bladder, as well as episodes of reflux pancreatitis, although it would permit, through the evaluation of the urinary concentration of amylases, an early diagnosis 
of organ rejection. Studies carried out at the Maryland prove that nowadays, thanks to new models of immunosuppressive therapy, a more a suitable antibiotic treatment, and the use of pancreatic biopsy for reject diagnosis and improved radiological techniques for the study of the transplanted pancreas, the enteric drain of the exocrine pancreatic secretions represents the golden standard [5].

\section{IMMUNOSUPPRESSIVE THERAPY}

A key point for the pancreas graft is the immunosuppressive therapy, which assume particular relevance due to the pronounced immunogenicity of this organ and the risk of recurrence of autoimmunity in patients affected by type 1 diabetes mellitus type 1 . The immunosupressive schemes more widely utilized today are based on the association of induction treatment (rATG, basiliximab, or alemtuzumab) and maintenance polytherapy (calcineurin inhibitors, cell proliferation inhibitors and steroids). Induction treatment has proved to be capable to reduce frequency and seriousness of rejection episodes and to improve renal function within 3 years from kidney-pancreas graft [6]. As far as maintenance therapy, it has been capital the transition from azathioprine to mycophenolate mofetil as inhibitor of cell proliferation, so as the definition of tacrolimus superiority compared to cyclosporine in the prevention of rejection in kidney-pancreas combined transplant [7] and the proof of the security of protocols with rapid decalage of steroids, with all the consequent advantages. The introduction of the present immunosupressive protocols has allowed to move from $78 \%$ pancreas rejection in the "cyclosporine era" to the current 5-25\%.

Immunosuppressants increase neoplastic risks, that should be carefully analyzed in a risk/benefit ratio evaluation before transplantation.

\section{OUTCOME AND RESULTS OF PANCREAS TRANSPLANT ON THE DIABETES COMPLICATIONS}

\section{Metabolic control}

Pancreas transplant restore near-normal glucose homeostasis, with a reduction of $\mathrm{HbA1}$ c levels (Fig. 1): in a cohort of 54 patients who received pancreas transplantation $\mathrm{HbA} 1 \mathrm{c}$ average after 6 years was $6 \%$, 
compared with $7 \%$ in patients who underwent intensive insulin therapy in Diabetes Control and Complications Trial.

Furthermore the pancreatic graft is able to restore glucagon secretion and consequently the blunted response to hypoglycemia, restore normal glucose hepatic production and improves lipid profiles.

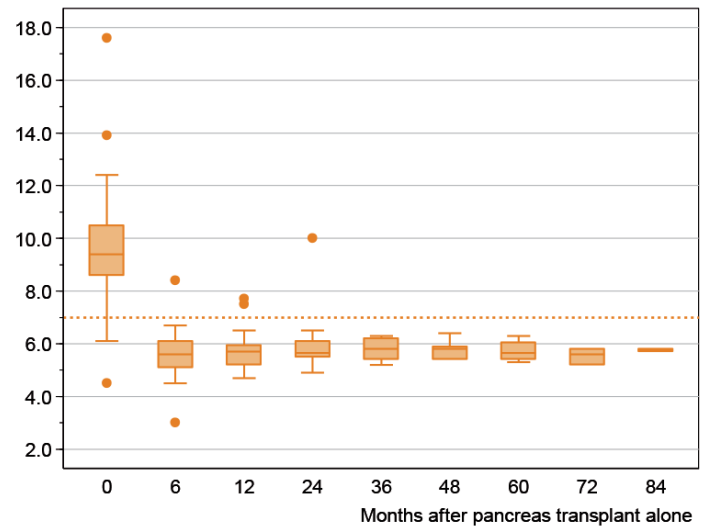

Fig. 1 - HbA1c levels after kidney-pancreas transplantation: a normalization is observed already at 6 months, and maintained over the years.

Retinopathy [8]

$75 \%$ of type 1 diabetes mellitus patients develop diabetic retinopathy within 10 years from the onsetof diabetes. Among these $30 \%$ presents proliferating retinopathy requiring laser therapyand $40 \%$ will suffer blindness within 3 years. Several studies show that, after initial worsening of diabetic retinopathy, in the long run pancreas transplantation positively affects the development of this invalidating complication of diabetes. A research realized by Chow to compare retinopathy profiles between patients submitted to well functioning kidney-pancreas simultaneous graft and patients who lost the pancreas, proves that $14 \%$ of patients will show an improvement in diabetic retinopathy, $76 \%$ remained stable, while $10 \%$ deteriorated. Nearly all the patients developed cataract, probably due to the immunosuppressive therapy, with a reduction of visual acuity. This study underlines the importance to obtain a stabilization of pre-transplant diabetic retinopathy in order to guarantee a non-progression of diabetic retinopathy. 
Nephropathy [9]

Hyperglycemia is a fundamental requirement for the development of the typical damages of the diabetic nephropathy. As shown by DCCT, patients affected by type 1 diabetes mellitus receiving an intensive insulin treatment, proved a reduced microalbuminuria incidence compared to patients receiving a standard treatment. Different studies have shown that achievement of normoglycemia is able to stop the worsening of diabetic nephropathy, but unable to recover when already present. Moreover other studies carried out by Fioretto et al. have proved that after 10 years of fully functional pancreatic graft, an improvement of glomerular and tubular damages was shown. Histology have shown reduction of the thickness of basal glomerular and tubular membranes and of mesengial matrix, as well as the disappearance of typical Kimmelstiel-Wilson nodular lesions. Additional studies have come to the conclusion that in the first stages the pancreas transplant leads to a significant decrease of proteinuria, obtaining a definite protection towards diabetic nephropathy.

Neuropathy [10]

Several studies carried out in patients submitted to simultaneous kidney-pancreas transplant (SPK) have proved the protective effect of normoglycemia on diabetic neuropathy. Kennedy et al. Have studied the progression of peripheral and autonomic neuropathy in pancreas transplanted patients compared to type 1 mellitus diabetic patients undergoing intensive insulin therapy. At 12, 24 and 42 months pancreas transplanted patients show a definite improvement in the sensorymotor and autonomic profile, whereas a worsening in the control group was evident. These observations were confirmed in other studies.

\section{Coronary heart disease [11]}

It is known that the high mortality rate in patients with type 1diabetes and end stage renal disease is mainly due to Ischemic Heart Disease. Jukema et al. have proved that at least $40 \%$ of patients undergoing pancreas transplantation show after transplantation a reduction of atherosclerotic damages, thus confirming previous observations of La Rocca et al. that demonstrated a reduction of carotid atherosclerosis 
in patients with a functioning simultaneous kidney-pancreas graft, when compared to a control group of patients with failed transplant. The Jukema study appears to point out that simultaneous kidney-pancreas transplant, although suffering major perioperatory side-effects, is able to reduce the coronaric atherosclerosis thanks to the euglycemia achieved, improving the prognosis and the quality of life of type1 mellitus diabetes patients. Furthermore metabolic alterations of type 1 diabetes lead to selective diastolic dysfunction: $30 \%$ of diabetic patients with cardiovascular disease show normal systolic function together with isolated diastolic dysfunction. This specific alteration was shown to be reversible 4 years after successful pancreas transplantation by the studies of La Rocca. Both La Rocca and Gaber also stress the positive impact on systolic function of the normalization of metabolic control in kidney-pancreas transplanted patients. An echocardiographic analysis within 2 years from kidney-pancreas transplant suggests an improvement of the left ventricle geometry in comparison with the same exam performed on matched patients who received only kidney transplant and therefore remained diabetic. Other studies have shown that the percentage of cardiac events like severe myocardial infarction and/or pulmonary edema are reduced in patients undergoing simultaneous kidney-pancreas transplant when compared to diabetic patients undergoing only kidney transplantation.

All these observations justify the inferior incidence of cardiovascular events and the higher rate of survival (see chapter Survival) in patients undergoing a kidney-pancreas transplant, when compared to diabetic patients undergoing kidney transplant alone.

\section{Cerebral and peripheral vasculopatby [12]}

Nankivell et al. has shown an initial worsening of carotid intima thickness in kidney-pancreas patients, without evidence of cerebrovascular accidents. More recent studies prove an improvement in the carotid intima thickness within 2 years from pancreas-kidney transplant independently from lipid profiles, body mass index, blood pressure control, smoke and use of antilipidic therapy. On the other hand, as to peripheral vasculopathy, a progressive worsening seems to occur, despite good pancreas transplant function. This behavior could be explained by a vascular damage, at the moment of transplantation, too advanced to be reversible. 
Survival [13]

SPK transplant was shown to increase, when compared to kidney transplant alone in diabetic patients (KTA), observed $v$ s. expected life span [8, 9] (Fig. 2). Data of the U.S. Scientific Renal Transplant Registry and of the U.S. Renal Data System (more than 13,000 patients studied) show that patient survival rate at 10 years was higher in SPK than in KTA, the former group showing greatest longevity (23.4 yrs) when compared to 20.9 yrs in KTA from living donors and 12.8 yrs in KTA from deceased donors [14].

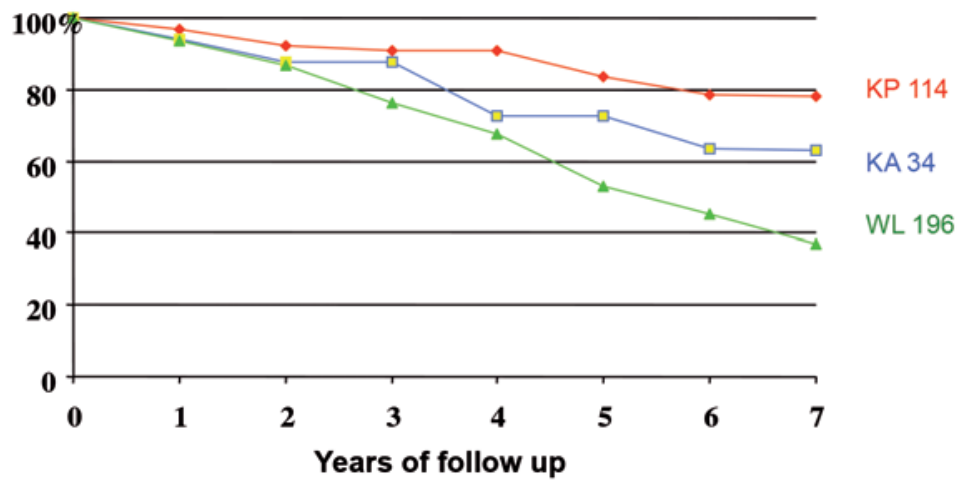

Fig. 2 - Actuarial survival in type 1 diabetic patients after Kidney-pancreas transplantation (KP, red), after Kidney transplantation alone (KA blue) and in waiting list for Kidney-pancreas transplantation (WL, green): pancreas transplantation improves live survival at 7 years of $18 \%$ over single kidney transplantation and of $40 \%$ over patents not yet transplanted.

Similar results were observed also in PAK patients, with an improvement of long term patient and kidney graft survival. When brittle diabetic patient are considered, the survival is higher at 4 years, when these patients receive a pancreas transplant, than in patients in waiting list for a transplant. In fact the survival percentage of patients included in the waiting list for pancreas transplant compared with patients undergoing transplant has been evaluated in a further UNOS data analysis. These results prove that within 4 years the survival rate in pancreas-transplanted patients vs. patients in waiting list for SPK reaches $90.3 \%$ (vs. $58.7 \%$ ) and for PAK $88.3 \%$ (vs. $81.7 \%$ ) and finally in the PTA group $90.5 \%$ (vs. $87.3 \%$ ). This study has confirmed that simultaneous kidney/pancreas transplant obtains survival global advan- 
tage in comparison with type1mellitus diabetes patients included in the waiting list for transplant [15].

From these data it appears that pancreas transplantation, that is not considered a life-saving procedure in the short term, must be considered a life-saving procedure in the long term.

SUMMARY BOX

- Pancreas transplantation is indicated in type 1 diabetic patients.

- Survival rates of patients and organs improved over years, achieving $95 \%$ and $85 \%$ respectively at 1 year.

- Pancreas transplantation normalize glucose metabolism

- Pancreas transplantation stabilize, reverts and prevent degenerative diabetic complications.

\section{ISLET TRANSPLANTATION}

Islet transplantation is a relatively new medical procedure aimed to replace pancreatic function. Unfortunately, the lack of standardized protocols and the differences in inclusion criteria and in immunosuppressive regimens among studies have prevented islet transplantation from becoming the gold standard treatment for patients affected by T1DM.

\section{INDICATIONS FOR ISLET CELL TRANSPLANTATION}

Frequent and severe hypoglycemic events are the most common indication for islet transplantation alone (ITA). Clinical and emotional problems associated with the use of exogenous insulin that are so severe as to be incapacitating, and consistent failure of insulin-based management to prevent acute complications are further indications for islet transplantation. Islet after kidney (IAK) transplantation is restricted to patients with end-stage renal disease affected by T1D previously submitted to successful kidney transplantation. A further group, rare, is simultaneous islet and kidney transplantation (SIK).

\section{PROCEDURE}

Islet transplantation is performed using pancreas from heart beating donors. 
After harvesting, the pancreas is processed in order to separate the islets from the surrounding tissue. This procedure is currently rather difficult with a relatively low rate of success (40-50\%). When al adequate islet preparation, in terms of quality and quantity, is available, it is generally infused within the liver, through a portography. The procedure can be performed under local anesthesia. Alternative sites were already proposed without success, so intrahepatic transplantation is currently the gold-standard procedure (Fig. 3).

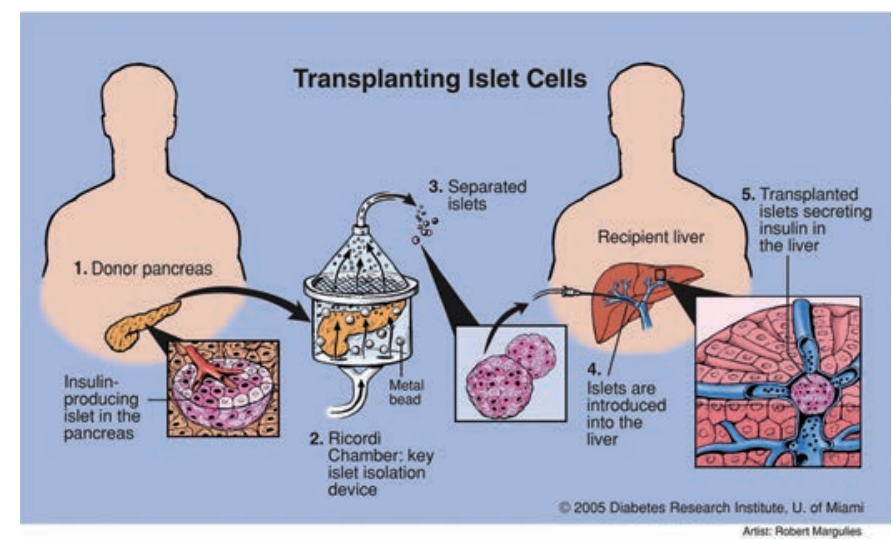

Fig. 3 -Schematic representation of islet transplantation.

The risks are minor and related to the portography procedure (bleeding, segmental liver thrombosis). Literature reports very limited side effects.

The procedure can be repeated when full function is not achieved.

\section{IMMUNOSUPPRESSION FOR ISLET TRANSPLANTATION}

As in any case of allotransplantation, islet transplantation requires immunosuppression in order to prevent allorejection. Furthermore islets seem to be more sensitive to recurrence of autoimmunity than pancreas transplantation, thus stressing the requirement of a complete and effective immunosuppression [16]. This constitutes the major risk for patients undergoing islet transplantation and should be carefully balanced according to the current 
clinical conditions of the patient and the potential advantages. Immunosuppressive schemes does not differ greatly from those used for pancreas transplantation. What characterize the immunosuppressive schemes in islet transplantation is the requirement of and antiinflammatory co-treatment, generally based on anti-TNF, aimed to prevent local inflammation at the transplant site, that greatly reduces islet engraftment. Immunosuppressants must be tightly monitored in order to avoid known nephrotoxic and diabetogenic effects. Furthermore Immunosuppressants increase neoplastic risks, that should be carefully analyzed in a risk/benefit ratio evaluation before transplantation.

\section{CLINICAL IMPACT OF ISLET TRANSPLANTATION}

Clinical results achievable with islet transplantation were fully analyzed by the Collaborative Islet Transplantation Registry (CITR) [17], held in Bethesda, that is collecting clinical data of the majority of the active Centers, since 1999. The registry has collected data of 677 patients who received islet transplantation alone (ITA) or islet transplantation after a successful kidney transplant (IAK) and compared results achieved in the periods 1999-2002, 2003-2006, 20072010. Insulin independence at 3 years was $27 \%$ in the first era $(214$ patients), $37 \%$ in the second era (255 pts) and $44 \%$ in the third era (208 pts), showing a progressive and constant improvement of the results.

When insulin independence is achieved all metabolic parameters improve (Fig. 4 and Fig. 5).

When full insulin independence is not achieved islet transplantation can in any case give an advantage to the patients by providing an auto regulated source of endogenous insulin, not enough to achieve full function, but enough to improve remarkably metabolic control with few units of insulin per day: this is considered "partial function" and is monitored though the blood levels of C-peptide, a surrogate marker of islet function. When C-peptide is higher than $0.3 \mathrm{ng} / \mathrm{ml}$ islet are considered still functioning, regardless of the insulin doses. This condition was maintained at 4 years in $60 \%$ in the first era, $74 \%$ in the second era and $88 \%$ in the third era, with a progressive and constant improvement also of this parameter of function. 


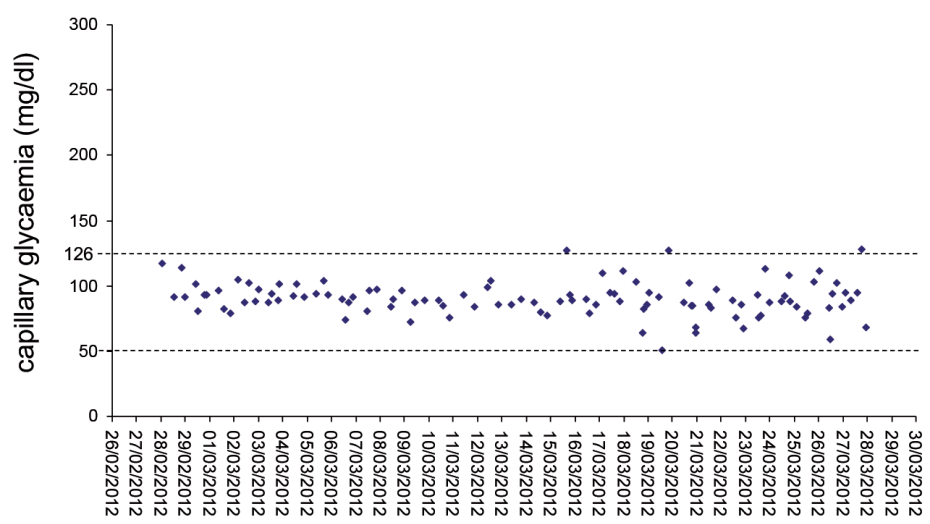

Fig. 4 -Blood glucose levels after islet transplantation are maintained within the normal range without insulin administration. These date are referred to a 40 years old type 1 diabetic patients, who developed diabetes at 19.

Insulin requirement was $0.38 \mathrm{U} / \mathrm{kg}$ pre transplantation and dropped to 0 after transplantation. $\mathrm{HbA1c}, 9.8 \%$ pre transplantation, was $5 \%$ after transplantation.

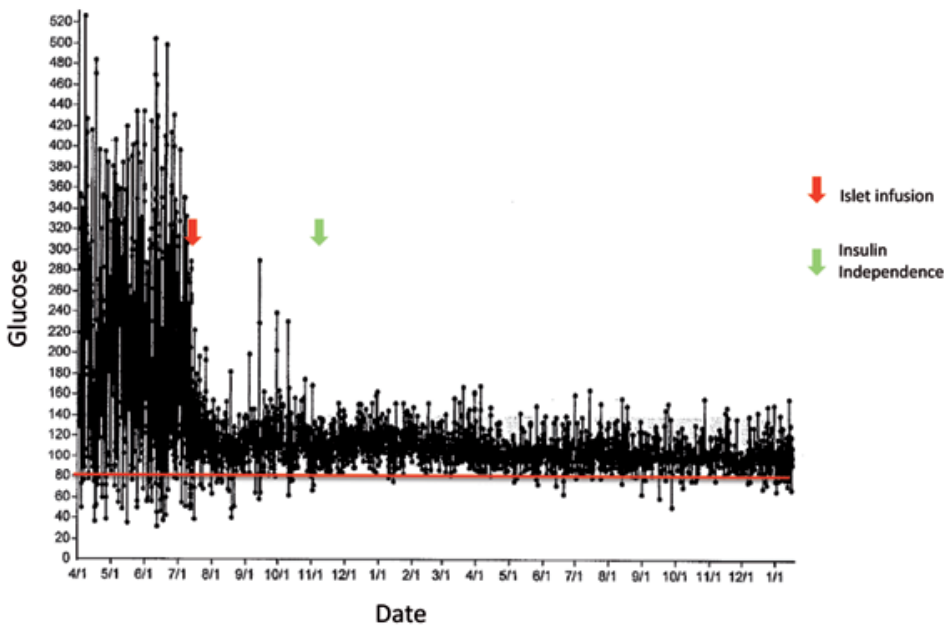

Fig. 5 - Blood glucose variability, very high before transplantation, is greatly reduced after transplantation.

As a consequence Glycated Hemoglobin improved over time and Hypoglycemic episodes nearly disappeared in patients showing islet function. 
A second islet infusion is required when full function is not achieved. Also this improved over years: $65 \%$ of patients required a second infusion in the era $1999-2006$, compared to $48 \%$ in the period 2007-2010.

\section{PATIENT SURVIVAL}

A study analyzed the effect of islet transplantation on patient survival [18]. This work compared two populations of kidney-islet transplanted patients: a successful group with C-peptide secretion $>0.5 \mathrm{ng} / \mathrm{mL}$ for more than 6 months and an unsuccessful group with early failure of the islet graft, who lost C-peptide secretion within 6 months of transplantation. All detailed studies before transplantation found that the two groups had similar general characteristics, metabolic status, immunosuppressive regimens, kidney graft function, degree of diabetic complications, and major known cardiovascular risk factors. After 7 years of follow-up, the survival among patients in the group with successful islet transplantation and sustained restoration of $\beta$-cell function was significantly higher $(90 \%)$ than among patients in the unsuccessful transplantation group (51\%). This higher survival in the successful group was accompanied by higher C-peptide levels and lower insulin requirements compared to the unsuccessful group, despite similar glycated hemoglobin levels. The number of cardiovascular deaths (according to ICD-9) was higher in the group with unsuccessful transplants, who also had poorer atherosclerotic profile and endothelial function [18].

\section{IMPACT OF ISLET TRANSPLANTATION ON DEGENERATIVE DIABETIC COMPLICATIONS}

Preliminary studies have shown that islet transplantation, even when not fully successful, can positively impact on late complications of diabetes, either preventing progression, either inducing a recover. The specific impact of islet transplantation on each of these complications is described in the following paragraphs. 


\section{MACROANGIOPATHY}

A previous report described the long-term beneficial effects of islet transplantation on micro- and macrovascular complications in 34 patients with T1D who received kidney transplants at a single institution. The authors found a reduction in carotid intima media thickness (IMT), an important index of eventual cardiovascular disease [18]. The increased IMT in the groups with nonfunctioning islets reached values higher than those in the general population but were similar to those in subjects with ischemic heart disease. Analysis of skin biopsies from islet-transplanted patients revealed that functioning islet transplants can induce positive micromodifications at the vessel level, such as an increase in the expression of von Willenbrand factor or endothelial nitric oxide or can reduce the thickening of the capillary basement membrane, cellular swelling, and the dilation of endoplasmic reticulum in endothelial cells [18]. The small sample size and single-center data render this study interesting but not definitive.

\section{KIDNEY FUNCTION}

Successful islet transplants in T1D patients with end-stage renal disease receiving kidney transplants is capable to prolong graft survival and prevent reduction in vascular function of the kidney graft [19]. Furthermore non-invasive assessments of graft vascular function using the Doppler resistance index and microalbuminuria evaluations showed that patients with T1D who received a kidney transplant and an islet transplant showed better renal vascular function and cumulative kidney graft survival than the group without functioning islet. The positive effect of islet transplantation on kidney function was confirmed by decreases in the urinary excretion of albumin, the urinary fractional excretion of sodium, and the sodium excretion rate.

\section{DIABETIC RETINOPATHY}

Little information is currently available on the effect of islet transplantation on diabetic retinopathy. A statistically significant increase in retinal blood flow velocity at 1 year in patients with T1D who received 
islet transplants [20] was reported (Fig. 6). The restoration of islet function can help control glucose excursion and, per se, can probably halt the alterations in retinal microcirculation. The Miami group reported a series of 12 patients who received islet transplants and were evaluated for the progression of diabetic retinopathy and neuropathy. Patients were examined by a single ophthalmologist and single neurologist throughout the study period. All patients showed stabilization of their retinopathic disease after islet transplantation.

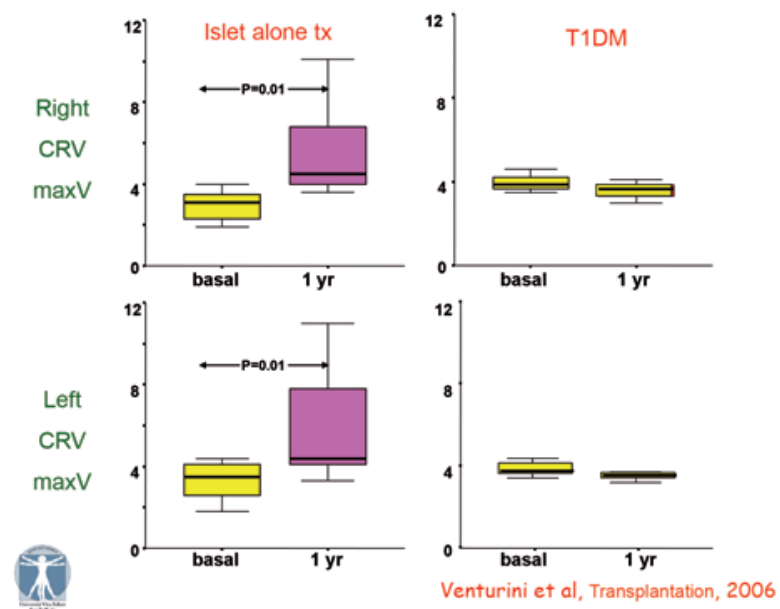

Fig. 6 - Right and left eye Central Retinal Velocity (CRV) in type 1 diabetic patients

1 year after islet transplantation (purple) and still in waiting list (yellow): a clear improvement is seen in the former group.

\section{DiABETIC NEUROPATHY}

Lee et al. assessed peripheral nerve function with a nerve conduction velocity (NCV) index [21) in islet-transplanted patients. Although no statistical analysis was provided, and the follow-up period was no longer than 2 years, the effect of $\beta$-cell replacement appeared to be positive on polyneuropathy. A preliminary report from the Milan group [22] showed, using the NCV index, that islet transplantation may induce long-lasting stabilization or even improvement of polyneuropathy in type 1 kidney-transplanted diabetic patients who also received a functioning islet transplant, reducing nerves' RAGE expression. 


\section{ISLET AUTOTRANSPLANTATION}

Islet autotransplantation (IAT) is a therapeutic approach used to prevent pancreatogenic diabetes or to reduce the severity of diabetes after a major pancreatectomy. This procedure was initially performed mainly in Minneapolis, staring at the end of seventies, the main indication being chronic painful panctreatitis. Indications were recently extended to pancreatectomy performed for the resection of benign tumors of the mid-segment of the pancreas or after total pancreatectomy for severe abdominal trauma. The aim of this procedure is to prevent post-pancreatectomy diabetes, that is very difficult to treat, due to the absence of counter-regulation effects of glucagon. Technically is very similar to islet allo-transplantation.

SUMMARY BOX

- Islet transplantation is indicated in type 1 diabetic patients.

- Success rate (insulin-independence) is around $80 \%$ at 1 year.

- Degenerative complications of diabetes can be halted by islet transplantation.

- Long term function is still fragile.

\section{WHEN PANCREAS AND WHEN ISLETS?}

A recent study has analyzed, in a single center study, the effects of pancreas and islet transplantation in an homogeneous population.

The outcomes of 33 PTA and 33 ITA were analyzed and pancreas or islet function (i.e., insulin independence), perioperative and longterm adverse events were recorded. A higher rate of insulin independence in PTA $(75 \%)$ versus ITA (59\%) wasobserved, with the longer insulin independence among PTA patients receiving tacrolimus. The burden of adverse events was higher for PTA patients in terms of hospitalization length and number, re-intervention for surgical and immunological acute complications, CMV reactivation and other infections.

This study sugests the practice of listing patients for PTA when the metabolic lability and the progression of chronic complications require a rapid normalization of glucose levels, with the exception of patients with cardiovascular disease, because of the high surgical risks. ITA is indicated when replacement of beta cell mass is needed in patients with a high surgical risk. 


\section{REFERENCES}

[1] Gruessner, A.C., Gruessner, R.W.G. Pancreas transplant outcomes for United States and Non United States cases as reported to the United Network for Organ Sharing and the International Pancreas Transplant Registry as of December 2011. Clinical Transplants 2012, Terasaki Foundation Laboratory, Los Angeles, CA.

[2] Stout RW. Insulin and atheroma. 20-yr perspective. Diabetes Care 1990; 13 : 631-54.

[3] Fontbonne A, Charles MA, Thibult N, et al. Hyperinsulinaemia as a predictor of coronary heart disease mortality in a healthy population: the Paris Prospective Study, 15-year follow-up. Diabetologia 1991; 34: 356-61.

[4] Hughes TA, Gaber AO, Amiri HS, et al. Kidney-pancreas transplantation. The eff ect of portal versus systemic venous drainage of the pancreas on the lipoprotein composition. Transplantation 1995; 60: 1406-1412.

[5] Kuo PC, Johnson LB, Schweitzer EJ, et al. Simultaneous pancreas/kidney transplantation-a comparison of enteric and bladder drainage of exocrine pancreatic secretions. Transplantation 1997; 63: 238-43.

[6] Burke et al. Prospective, randomized trial of the effect of antibody induction in simultaneous pancreas and kidney transplantation: three-year results. Transplantation (2004) vol. 77 (8) pp. 1269-75.

[7] Malaise et al. Tacrolimus compared with cyclosporine microemulsion in primary simultaneous pancreas-kidney transplantation: the EURO-SPK 3-year results. Transplant Proc (2005) vol. 37 (6) pp. 2843-5.

[8] Chow VC, Pai RP, Chapman JR, et al. Diabetic retinopathy after combined kidney-pancreas transplantation. Clin Transplant 1999; 13: 356-62.

[9] Fioretto P, Steffes MW, Sutherland DE, et al. Reversal of lesions of diabetic nephropathy after pancreas transplantation. N Engl J Med 1998; 339: 69-75.

[10] Martinenghi S, Comi G, Galardi G, et al. Amelioration of nerve conduction velocity following simultaneous kidney/pancreas transplantation is due to the glycaemic control provided by the pancreas. Diabetologia 1997; 40: 1110-11.

[11] Jukema JW, Smets YF, van der Pijl JW, et al. Impact of simultaneous pancreas and kidney transplantation on progression of coronary atherosclerosis in patients with end-stage renal failure due to type 1 diabetes. Diabetes Care 2002; 25: 906-11.

[12] Fiorina P, La Rocca E, Venturini M, et al. Effects of kidney-pancreas transplantation on atherosclerotic risk factors and Endothelial Function in Type-1 diabetic uremic patients. Diabetes, 50: 496-501.

[13] La Rocca E, Fiorina P, Di Carlo V, et al. Cardiovascular outcomes after kidneypancreas and kidney-alone transplantation. Kidney Int 2001;60: 1964-71.

[14] Reddy, K.S., Stablein, D., Taranto, S. Long-term survival following simultaneous kidney-pancreas transplantation versus kidney transplantation along in patients with type-1 diabetes mellitus and renal failure. Am J Kidney Dis 2003; 41:464-470.

[15] Gruessner RW, Sutherland DE, Gruessner AC. Mortality assessment for pancreas transplants. Am J Transplant 2004; 4: 2018-26. 
[16] Piemonti, L; Everly, ; Maffi, P; et al. Alloantibody and autoantibody monitoring predicts islet transpnatation outcome in human type 1 diabetes. Diabetes. 2013 May;62(5):1656-64.

[17] Barton $\mathrm{F}$ et al. Improving outcomes in clinical islet transplantation: 1999-2010. Diabetes Care 35:1436-1445, 2012.

[18] Fiorina P, Gremizzi C, Maffi P, et al. Islet transplantation is associated with an improvement of cardiovascular function in type 1 diabetic kidney transplant patients. Diabetes care 2005;28(6):1358-1365.

[19] Fiorina P, Venturini M, Folli F, et al. Natural history of kidney graft survival, hypertrophy, and vascular function in end-stage renal disease type 1 diabetic kidney-transplanted patients: beneficial impact of pancreas and successful islet cotransplantation. Diabetes care 2005;28(6):1303-1310.

[20] Venturini M, Fiorina P, Maffi P, et al. Early increase of retinal arterial and venous blood flow velocities at color Doppler imaging in brittle type 1 diabetes after islet transplant alone. Transplantation 2006;81(9):1274-1277.

[21] Lee TC, Barshes NR, O'Mahony CA, et al. The effect of pancreatic islet transplantation on progression of diabetic retinopathy and neuropathy. Transplant Proc 2005;37(5):2263-2265.

[22] Del Carro U, Fiorina P, Amadio S, et al. Evaluation of polyneuropathy markers in type 1 diabetic kidney transplant patients and effects of islet transplantation: neurophysiological and skin biopsy longitudinal analysis. Diabetes care 2007;30(12):3063-3069. 